\title{
Minimal attractors and bifurcations of random dynamical systems
}

\author{
By PETER AshWIN \\ Department of Mathematics and Statistics, University of Surrey, \\ Guildford GU2 $5 X H, U K$ \\ Received 20 August 1997; accepted 24 November 1998
}

We consider attractors for certain types of random dynamical systems. These are skew-product systems whose base transformations preserve an ergodic invariant measure.

We discuss definitions of invariant sets, attractors and invariant measures for deterministic and random dynamical systems. Under assumptions that include, for example, iterated function systems, but that exclude stochastic differential equations, we demonstrate how random attractors can be seen as examples of Milnor attractors for a skew-product system. We discuss the minimality of these attractors and invariant measures supported by them.

As a further connection between random dynamical systems and deterministic dynamical systems, we show how dynamical or D-bifurcations of random attractors with multiplicative noise can be seen as blowout bifurcations, and we relate the issue of branching at such D-bifurcations to branching at blowout bifurcations.

Keywords: chaotic dynamics; random dynamical systems; forced systems; blowout bifurcation

\section{Introduction}

A random dynamical system (RDS) has two ingredients: (i) a model of the noise in the form of a measure-preserving transformation; and (ii) a model of the deterministic dynamics consisting of a family of continuous transformations. Which transformation is applied depends on the state of the noise at that moment in time. We examine RDSs generated by iterating skew-product maps of the form

$$
F(w, x)=\left(\theta(w), \psi_{w}(x)\right) .
$$

We say $\theta$ is the base transformation (the noise process) and $\psi$ is the fibre transformation (the forced system). We write $\psi_{w}(x)$ rather than $\psi(w, x)$ for convenience of notation.

We assume that $\theta$ preserves an ergodic invariant measure $\mathcal{P}$ (we are only interested in 'stationary noise') and $\psi$ is continuous in $x$. The theory of such systems has been developed over the last 15 years as a way to model systems that are forced by random perturbations. For an overview, see Arnold (1998) and references therein.

Many results have been obtained relating to attractors and invariant measures in such systems; this description has advantages over a Fokker-Planck approach in that it retains details of the dynamics of the system, and, moreover, is valid for noise of any magnitude. The Fokker-Planck stationary probability densities only give

Proc. R. Soc. Lond. A (1999) 455, 2615-2634

Printed in Great Britain 
information about the asymptotic distribution of the trajectory rather than how it moves around the distribution; they also do not give information about 'unstable' trajectories, which can be obtained from study of RDSs. Recent work on RDSs has given definitions for 'random attractors' and has studied how these can lose stability at so-called dynamic or D-bifurcations.

Motivated by totally different questions (notably by synchronization of coupled dynamical systems (see, for example, Ashwin et al. 1994)), several researchers have studied continuous iterated maps of the form,

$$
F(x, y)=(f(x)+h(x, y), g(x, y))
$$

for $x \in \boldsymbol{R}^{n}, y \in \boldsymbol{R}^{m}$ where $h(x, 0) \equiv g(x, 0) \equiv 0$, and have made a number of interesting observations (note that $h(x, y) \equiv 0$ gives a skew-product system). Firstly, one can quite robustly find examples of attractors in (1.2) that have so-called riddled basins, i.e. their basins have positive Lebesgue measure but contain no open sets (Alexander et al. 1992). These attractors are only attractors in the sense of Milnor (1985). Secondly, chaotic attractors for (1.2) in $y=0$ typically lose stability at what has been called a blowout bifurcation of chaotic attractors (Ott \& Sommerer 1994).

In this paper we show that for many RDSs, random attractors (respectively Dbifurcations for multiplicative noise) can be related to Milnor attractors (respectively blowout bifurcations) of (1.1); there are deep connections between the two types of system and we speculate that much progress can be made by transfer of techniques and results between them.

We will assume that the base transformation $\theta$ is continuous on some compact metric space, and that the fibre transformations $\phi$ are continuous in both variables. This is not usually assumed for RDSs (only measurability is usually assumed), but it is valid for a number of important applications and this assumption gives one a lot of control over the structure of attractors.

The rest of this paper is set out as follows: in $\S 2$ we describe attractors, Milnor attractors, invariant measures and define random dynamical systems. Section 3 discusses definitions of random invariant sets and random attractors for RDSs and relates them to invariant sets and attractors of the skew-product dynamical system. We suggest a definition of a random attractor using the ideas of Milnor for deterministic systems. We define minimal attractors (i.e. those that appear as $\omega$-limit sets for large sets of initial conditions) rather than concentrating on global attractors (cf. Crauel \& Flandoli 1994; Schenk-Hoppé 1996a,b). In $\S 4$ we discuss random invariant measures, introduce the idea of random natural measure, and discuss some exceptional behaviour of trajectories characterized by what we refer to as inner singular measures.

Section 5 considers random dynamical systems with multiplicative noise. We use an elementary example to illustrate a number of connections between RDSs and deterministic dynamical systems. We use Lyapunov exponents to classify the stability of the trivial random invariant set and give an example of the sort of random attractor that results from bifurcation. We show how a D-bifurcation of a random attractor in this example can be seen as a deterministic blowout bifurcation in the skew-product system.

Section 6 briefly discusses the results and speculates on extensions and generalizations. Throughout, we concentrate on smooth discrete-time RDSs to keep arguments as simple as possible because we aim to point out connections rather than give optimal or totally general results.

Proc. R. Soc. Lond. A (1999) 
Notation and conventions All measures discussed are Borel measures of a metric space $M$ with metric $d_{M}$, i.e. they are measurable with respect to the Borel $\sigma$ algebra generated by the closed subsets of $M$. If $A$ and $B$ are subsets of $M$, then we write $d_{M}(x, B)=\inf _{y \in B} d_{M}(x, y)$ and $d_{M}(A, B)=\sup _{x \in A} \inf _{y \in B} d_{M}(x, y)$. Note that $d_{M}(A, B)=0$ implies that $A \subseteq \bar{B} . h(A, B)=d_{M}(A, B)+d_{M}(B, A)$ is the Hausdorff metric on compact subsets of $M$. We define

$$
\operatorname{Pr}(M)=\{\text { Borel probability measures on } M\},
$$

i.e. measures with $\mu(M)=1$. This can be endowed with the weak-* topology defined by $\mu_{n} \rightarrow \mu$ if and only if

$$
\int f \mathrm{~d} \mu_{n} \rightarrow \int f \mathrm{~d} \mu
$$

for all bounded continuous $f$ (limits as $n \rightarrow \infty$ ). In fact, if $M$ is a Polish space (a separable metric space with complete metric), then this topology on $\operatorname{Pr}(M)$ is also metrizable (see, for example, Dudley 1987).

\section{Attractors and invariant measures}

We recall some standard concepts of attractor for deterministic (i.e. non-random) dynamical systems. Let

$$
\theta: W \rightarrow W
$$

be a continuous map of a topological space $W$ to itself. Given an initial point $w_{0} \in W$, consider the trajectory,

$$
\left\{w_{0}, \theta\left(w_{0}\right), \theta^{2}\left(w_{0}\right), \ldots\right\},
$$

obtained by iterating the map and define the $\omega$-limit set

$$
\omega\left(w_{0}\right)=\left\{\text { limits of } \theta^{n}\left(w_{0}\right) \text { as } n \rightarrow \infty\right\}=\bigcap_{N>0} \overline{\bigcup_{n>N} \theta^{n}\left(w_{0}\right)} .
$$

In general, an attractor for a deterministic dynamical system is a closed invariant subset of the phase space to which most of the trajectories are asymptotic; we will only deal with attractors that are compact for convenience. We need to define what is meant by most and asymptotic in the previous sentence; different definitions give rise to a plethora of different concepts of attractor.

We say a closed invariant set $A$ is an asymptotically stable attractor if for any neighbourhood $U$ of $A$ there is a neighbourhood $V$ of $A$ such that $\theta^{n}(V) \subseteq U$ for all $n \in \boldsymbol{N}$ and

$$
A=\bigcap_{n \in N} \theta^{n}(V) .
$$

In this case, clearly all initial points in $U$ have $\omega$-limit sets contained in $A$.

A global attractor is the smallest closed connected invariant set $A$ such that every initial condition is asymptotic to it, i.e.

$$
A \subseteq \bigcup_{w \in W} \omega(w) .
$$

A minimal attractor contains no proper subsets that are also attractors. Given a global attractor, one needs to find all minimal attractors on this global attractor in

Proc. R. Soc. Lond. A (1999) 
order to say what a typical $\omega$-limit set will look like. Most of a global attractor is only seen in the $\omega$-limits of exceptional (if any) trajectories.

For more complicated or chaotic deterministic dynamics it is necessary to take measures into account to get a useful description of the dynamics. We assume that there is a 'background measure' $\ell(\cdot)$ and that we will only choose initial conditions from an $\ell$-full measure subset. If there is an ergodic measure $\mu$ that determines the statistics of forward trajectories of a non-zero measure set of initial conditions with respect to $\ell$, we say $\mu$ is a natural measure (see, for example, Ruelle 1989; Eckmann \& Ruelle 1985) (Sinai-Bowen-Ruelle measures) are natural measures that are absolutely continuous on unstable manifolds). Any ergodic invariant measure that is absolutely continuous with respect to $\ell$ is clearly a natural measure. Although a lot of effort has gone into finding such measures for deterministic dynamical systems, existence has only been shown for relatively few systems (see, for example, Mora \& Viana 1993). For a thorough survey of the ergodic theory of continuous dynamical systems, we refer the reader to the book of Katok \& Hasselblatt (1995).

An example of some of the complexities of chaotic attractors can be seen in the doubling map $x \mapsto 2 x \bmod 1$ on the unit interval. This has uncountably many ergodic invariant measures supported in the interval $[0,1]$; even restricting to those ergodic invariant measures whose support is the whole of $[0,1]$ there are uncountably many such singular measures isomorphic to Bernoulli shifts $B(p, 1-p), p \in(0,1)$. If we take the Lebesgue measure as the background measure, we find that the Lebesgue measure is itself a natural measure for the transformation, and the only minimal attractor is the whole interval. For more typical transformations, natural measures (if they exist) will be supported only on a subset of the phase space.

\section{(a) Milnor attractors}

Suppose we iterate (2.1) with $W$, a compact metric space, and we have a background measure $\ell_{W}$ on $W$ that assigns positive measure to open sets. Suppose that $A$ is a (non-empty) compact invariant subset of $W$. We define the basin (or realm) of attraction of $A$

$$
\mathcal{B}(A)=\{w \in W: \omega(w) \subseteq A\} .
$$

Definition 2.1. A compact invariant set $A$ is an attractor if $\ell(\mathcal{B}(A)) \neq 0$. If $A$ contains no proper closed invariant subset $A^{\prime}$ with $\ell\left(\mathcal{B}(A) \backslash \mathcal{B}\left(A^{\prime}\right)\right) \neq 0$, then we say $A$ is a minimal attractor.

This definition is slightly different to Milnor (1985) in that he defines a stronger notion of minimality, namely that there is no proper closed invariant subset $A^{\prime}$ with $\ell\left(\mathcal{B}\left(A^{\prime}\right)\right) \neq 0$. An asymptotically stable attractor is an attractor in this sense but not vice versa. If $\mathcal{B}(A)$ contains an open neighbourhood, then $A$ is an attractor. The riddled basin examples of Alexander et al. (1992) give apparently robust examples of attractors whose basins have positive measure but open dense complement.

\section{(b) Natural measures}

We define the set of $\theta$-invariant probability measures

$$
\underset{\text { inv }}{\operatorname{Pr}}(W)=\{\mu \in \operatorname{Pr}(W): \theta \mu=\mu\}
$$

Proc. R. Soc. Lond. A (1999) 
and

$$
\mathcal{E}(W)=\{\text { ergodic measures in } \underset{\text { inv }}{\operatorname{Pr}}(W)\},
$$

that is, the set of $\mu \in \operatorname{Pr}_{\text {inv }}(W)$ such that all $\theta$-invariant sets have zero or full $\mu$-measure. By the ergodic decomposition theorem (see, for example, Katok \& Hasselblatt 1995, theorem 4.1.12), this is the set of extremal points of $\operatorname{Pr}_{\text {inv }}(M)$. Now consider the orbit through a point $w \in W$; we define a sequence of empirical measures in $\operatorname{Pr}(W)$ by

$$
m_{n}(w)=\frac{1}{n} \sum_{k=0}^{n-1} \delta_{\theta^{k}(w)}
$$

(see, for example, Ruelle 1989), and let

$$
\omega_{\text {meas }}(w)=\left\{\text { limits of } m_{n} \text { as } n \rightarrow \infty\right\}
$$

within $\operatorname{Pr}(W)$. Finally, for any $\mu \in \mathcal{E}(W)$, we define the generic set for the measure $\mu$ to be

$$
\mathcal{G}(\mu)=\left\{w \in W: \omega_{\text {meas }}(w)=\mu\right\},
$$

i.e. all points whose asymptotic statistics are governed by $\mu$. Note that Birkhoff's ergodic theorem implies that $\mathcal{G}(\mu) \cap \operatorname{supp}(\mu)$ has full $\mu$-measure for all $\mu \in \mathcal{E}(W)$.

Definition 2.2. If $\ell(\mathcal{G}(\mu)) \neq 0$, then we say $\mu \in \mathcal{E}(W)$ is a natural measure for (2.1).

Observe the similarity between this and the definition of an attractor. As discussed in Ashwin (1997), if $A=\operatorname{supp}(\mu)$ is compact and $\ell(\mathcal{G}(\mu)) \neq 0$, it is still possible to have $\ell(\mathcal{B}(A))=0$.

\section{(c) Inner singular invariant measures}

If an ergodic invariant measure has a zero measure generic set, this does not necessarily mean that we cannot approximate it using orbits of points that are generic for another measure. More precisely, suppose that $\mu \in \mathcal{E}(W)$ is an ergodic measure and define

$$
\underset{\mu}{\operatorname{Pr}}=\{\nu \in \operatorname{Pr}(W): \operatorname{supp}(\nu) \subseteq \operatorname{supp}(\mu)\}
$$

and

$$
\mathcal{E}_{\mu}=\{\text { ergodic measures in } \underset{\mu}{\operatorname{Pr}}\} .
$$

Since $\mu$-almost all trajectories will spend arbitrarily long times near the support of measures in $\mathcal{E}_{\mu}$, it is reasonable to expect that this set will affect the long-time behaviour of the system in some way. We refer to the set

$$
\mathcal{E}_{\mu} \backslash\{\mu\},
$$

as the set of inner singular measures with respect to $\mu$. If there are inner singular measures, we refer (rather unconventionally) to the dynamics on $\operatorname{supp}(\mu)$ as chaotic.

The inner singular measures make themselves apparent through the dynamics in the following way. Consider

$$
m_{k, l}(w)=\frac{1}{k-l} \sum_{j=l}^{k-1} \delta_{\theta^{j}(w)}(\cdot),
$$

Proc. R. Soc. Lond. A (1999) 
and define (cf. (2.3))

$$
\tilde{\omega}_{\text {meas }}(w)=\left\{\text { limits of } m_{k, l} \text { as } k-l \rightarrow \infty\right\} .
$$

Note that $\omega_{\text {meas }}(w) \subseteq \tilde{\omega}_{\text {meas }}(w)$.

Lemma 2.3. For all $w \in \mathcal{G}(\mu)$, we have

$$
\mathcal{E}_{\mu} \subseteq \tilde{\omega}_{\text {meas }}(w) .
$$

Proof. Pick any $\nu \in \mathcal{E}_{\mu}$, any $z \in \mathcal{G}(\nu) \cap \operatorname{supp}(\nu)$, and let $z_{n}=\theta^{n}(z)$. Now consider any $w \in \mathcal{G}(\mu)$; it is clear that $\omega(w) \supseteq \operatorname{supp}(\mu)$. Let $w_{n}=\theta^{n}(w)$ and choose a monotonic increasing sequence of $n_{i}$ such that $w_{n_{i}} \rightarrow z$ as $i \rightarrow \infty$. Given any continuous observable $f: W \rightarrow \boldsymbol{R}$ and any $N>1$, continuity of $\theta$ implies that

$$
\sup _{1 \leqslant j \leqslant N}\left|f\left(w_{n_{i}+j}\right)-f\left(z_{j}\right)\right| \rightarrow 0
$$

as $i \rightarrow \infty$. Hence,

$$
\lim _{j \rightarrow \infty} \lim _{i \rightarrow \infty} m_{n_{i}, n_{i}+j}(w)=\nu
$$

and so $\nu \in \tilde{\omega}_{\text {meas }}(w)$.

Note that $\tilde{\omega}_{\text {meas }}(w)$ is contained in the convex hull of $\mathcal{E}_{\mu}$. We say that trajectories in $\mathcal{G}(\mu)$ shadow the generic sets of all inner singular measures with respect to $\mu ; \theta$ needs to be continuous for this shadowing to occur. If $\mu$ is a natural measure, then (2.4) will hold for a set of $w$ with non-zero $\ell$-measure.

\section{(d) Continuous RDSs}

We will concentrate on the case of continuous RDSs in a sense that we make precise below. More general cases, such as where an RDS is generated by a random differential equation or a stochastic differential equation, will introduce a host of technical problems without contributing to the ideas we want to present here. For example, in terms of Arnold (1998), we deal only with discrete time RDSs that are $C^{\infty}$ and have extra smoothness assumptions. Nonetheless, many of the following ideas can, presumably, be generalized to other settings, as long as there is continuity in base variables.

We make the following standing assumptions.

(H1) $X$ is a compact region in $\boldsymbol{R}^{n}$ and $\ell$ is a Lebesgue equivalent measure on $X$.

(H2) $W$ is a compact metric space with metric $d_{W}(\cdot, \cdot)$, and $\theta: W \rightarrow W$ is a homeomorphism that preserves an ergodic probability measure $\mathcal{P} \in \mathcal{E}(W)$. This measure assigns positive measure to open sets in $W$.

(H3) $\psi: W \times X \rightarrow X$ is continuous in both arguments and $\psi_{w}$ is a homeomorphism for each $w$.

Let $l(\cdot)$ be the background measure on $W \times X$ generated by $l(A \times B)=\mathcal{P}(A) \times \ell(B)$ for measurable $A \subset W, B \subset X$. The map

$$
F: W \times X \rightarrow W \times X
$$

Proc. R. Soc. Lond. A (1999) 
defined by (1.1), generates a dynamical system upon iteration. The result of $n$ iterations of $F$ can be written

$$
F^{n}(w, x)=\left(\theta^{n}(w), \phi(n, w) x\right),
$$

where $\phi: Z \times W \times X \rightarrow X$ is a cocycle over the map $\theta$, and one can compute

$$
\begin{aligned}
\phi(n, w) x & =\psi_{\theta^{n-1}(w)} \circ \cdots \circ \psi_{w}(x), \\
\phi(0, w) x & =x, \\
\phi(-n, w) x & =\psi_{\theta^{-n+1}(w)}^{-1} \circ \cdots \circ \psi_{\theta^{-1}(w)}^{-1}(x),
\end{aligned}
$$

for all $n \in\{1,2,3, \ldots\}, w \in W$ and $x \in X . \dagger$

Definition 2.4. We refer to the cocycle $\phi$ in (2.5) as a random dynamical system or RDS over the transformation $\theta$.

Similar systems are studied in periodically, quasiperiodically and chaotically forced systems (see, for example, Stark 1997), and in the context of iterated function systems (see, for example, Kifer 1988; Falconer 1997). If the cocycle has trivial dependence on $w$, then it defines a (true) dynamical system on $X$; if it is non-trivial, then many of the ideas of dynamical systems can be reformulated in an $w$-dependent way.

\section{Random invariant sets and attractors}

A random set is a map $A: W \rightarrow 2^{X}$, i.e. a set of subsets of $X$ indexed by $W$, such that $A$ is $\mathcal{P}$-measurable. A closed random set is such that $A(w)$ is closed for $\mathcal{P}$-almost all $w$. There is an equivalence relation on random sets by ignoring differences on subsets with $\mathcal{P}$-measure zero; there is a partial ordering $A \subseteq$ as $B$ on closed random sets defined by $A \subseteq B$ if and only if $A(w) \subseteq B(w)$ for $\mathcal{P}$-almost all $w$.

Definition 3.1. A closed non-empty random set $A$ is said to be (forward) invariant under the RDS (1.1) if

$$
\psi_{w} A(w) \subseteq A(\theta(w)),
$$

i.e. if $d_{X}\left(\psi_{w} A(w), A(\theta(w))\right)=0$ for $\mathcal{P}$-almost all $w$. It is exactly invariant if

$$
\psi_{w} A(w)=A(\theta(w)) .
$$

If $A$ is a random invariant set, it attracts a random set $B$ (and write $B \rightarrow A$ ) if

$$
d_{X}\left(\phi\left(n, \theta^{-n}(w)\right) B\left(\theta^{-n}(w)\right), A(w)\right) \rightarrow 0,
$$

as $n \rightarrow \infty$ for $\mathcal{P}$-almost all $w$ (Crauel \& Flandoli 1994). The random $\omega$-limit set of a random set $B$ is defined to be the random set

$$
\omega_{\text {rand }}(B)(w)=\bigcap_{N>0} \overline{\bigcup_{n>N} \phi\left(n, \theta^{-n}(w)\right) B\left(\theta^{-n} w\right)} .
$$

Remark 3.2. Note that the $\omega$-limit of a single point $(w, x) \in W \times X$ is empty unless $w$ is a periodic point for $\theta$. This is one of the main difficulties of defining a random attractor without putting a topology on $W$; one needs to consider $\omega$-limits of random sets as above. We give a different definition using instead the metric on $W$.

$\dagger$ We use $w \in W$ rather than $\omega \in \Omega$ to denote the random forcing variable; this is to keep a similarity with the RDS literature while using $\omega$ for its conventional deterministic use.

Proc. R. Soc. Lond. A (1999) 
Definition 3.3. Suppose $A$ is a random invariant set and define the random basin of attraction of $A$ to be the random set

$$
\mathcal{B}(A)(w)=\left\{x \in X \text { such that } d_{X}\left(\phi(n, w) x, A\left(\theta^{n}(w)\right)\right) \rightarrow 0 \text { as } n \rightarrow \infty\right\} .
$$

Remark 3.4. We compare this 'pointwise' definition with that of Crauel \& Flandoli (1994) and Schenk-Hoppé (1996a) who define the random domain of attraction to be the universe of sets $B$ such that $B \rightarrow A$. Suppose we have a $B$ in this random domain; this implies (see, for example, Crauel \& Flandoli 1994, lemma 3.4) that

$$
d_{X}\left(\phi(n, w) B(w), A\left(\theta^{n}(w)\right)\right) \rightarrow 0,
$$

as $n \rightarrow \infty$, where convergence is in probability. If $B(w)$ were such that convergence (3.1) is almost sure, then $B(w) \subseteq \mathcal{B}(A)(w)$.

If we replace almost sure convergence by convergence in probability in the definition of random basin, we speculate that this corresponds to a definition for a random essential basin of attraction (see Ashwin et al. 1998).

A random neighbourhood of a random set $A$ is defined to be a random set $N(w)$ such that for $\mathcal{P}$-a.a. $w \in W, N(w)$ is a neighbourhood of $A(w)$ in $X$.

Schenk-Hoppé $(1996 a, b)$ discussed a definition of a random attractor in terms of a random invariant set that attracts a random neighbourhood (see also Crauel \& Flandoli 1994). This suggests the following definition.

Definition 3.5. A random compact invariant set $A(\omega)$ is a random attractor if $\mathcal{B}(A)$ contains a random neighbourhood of $A$.

A stronger concept of attraction is that of uniform stability where the neighbourhood can be chosen independent of $w$.

Definition 3.6. A random compact invariant set $A$ is uniformly stable if $\mathcal{B}(A)$ contains a (non-random) neighbourhood of $A$.

In this case, the neighbourhood is independent of $w$. Note that for a stochastic differential equation, the only non-random neighbourhood containing $A(w)$ will typically be the whole space $X$. Such stability can, however, be realized in RDSs with bounded noise.

We endow $W \times X$ with the product topology and metric

$$
d((v, x),(w, y))=d_{W}(v, w)+d_{X}(x, y) .
$$

For any $A: W \rightarrow 2^{X}$, we define $\operatorname{graph}(A) \subset W \times X$ as the subset

$$
\operatorname{graph}(A)=\{(w, x): x \in A(w)\} .
$$

(a) Relating random invariant sets and $F$-invariant sets

If $A$ is an invariant random set, then $\operatorname{graph}(A)$ is not necessarily defined, as $A$ is an equivalence class. However, the (essential) closure of $\operatorname{graph}(A)$

$$
\overline{\operatorname{graph}(A)}=\bigcap_{B} \overline{\operatorname{graph}(B)},
$$

where we take the intersection over all $B: W \rightarrow 2^{X}$ with $B(w)=A(w)$ for $\mathcal{P}$-a.a. $w$ (the closure is relative to the topology on $W \times X$ ). We define $\tilde{A}(w)$ by

$$
\operatorname{graph}(\tilde{A})=\overline{\operatorname{graph}(A)} \text {. }
$$

Proc. R. Soc. Lond. A (1999) 
This is a set-valued function defined on $w \in \operatorname{supp}(\mathcal{P})$. It will, in general, be much larger than the fibrewise closure of $A(w)$ (cf. Schenk-Hoppé 1996b). In fact, one can have

$$
\operatorname{dim}(\tilde{A}(w))>\operatorname{dim}(A(w))
$$

almost everywhere. Since we assume that $X$ is compact, $\overline{\operatorname{graph}(A)}$ is also compact.

An alternative but equivalent characterization of $\tilde{A}$ is given by the following lemma.

Lemma 3.7. Suppose that $A$ is a random set. Then $\tilde{A}(w)$ is the set of $x$ such that given any $S \subset W$ with $\mathcal{P}(S)=1$, there is a sequence $\left\{w_{i}\right\} \subset S$ and $x_{i} \in A\left(w_{i}\right)$ such that $\left(w_{i}, x_{i}\right) \rightarrow(w, x)$.

This expresses the closure explicitly in terms of limits.

Lemma 3.8. Suppose that $A(w)$ is a random invariant set. Then $\overline{\operatorname{graph}(A)}$ is F-invariant.

Proof. By invariance of $A$ we know that

$$
d_{X}\left(\psi_{w} A(w), A(\theta(w))\right)=0
$$

for $\mathcal{P}$-a.a. $w$. Moreover, if

$$
d_{X}\left(\psi_{w_{i}} x_{i}, A\left(\theta\left(w_{i}\right)\right)\right)=0
$$

for each $i$, then by continuity of $\psi$ and $\theta$, any limit $\left(w_{i}, x_{i}\right) \rightarrow(w, x)$ will satisfy

$$
d_{X}\left(\psi_{w} x, \tilde{A}(\theta(w))\right)=0 .
$$

Thus $\tilde{A}$ and, hence, $\overline{\operatorname{graph}(A)}$ are invariant.

Remark 3.9. The set $A(w)$ has properties that depend on the dynamics $\theta$ as well as $\phi$. In particular, if $\theta$ is uniquely ergodic, then $A(w)$ may be continuous. In the chaotic case (or if $\theta$ or $\psi$ are discontinuous), $A(w)$ will typically only be measurable in $w$.

\section{(b) Random Milnor attractors}

We propose a (weaker) concept than definition 3.5 inspired by definition 2.1; this uses the background measure on $X$.

Definition 3.10. A random compact invariant set is a random Milnor attractor if

$$
\ell(\mathcal{B}(A)(w)) \neq 0
$$

for $\mathcal{P}$-almost all $w$. It is a minimal random Milnor attractor if there is no random compact invariant set $A^{\prime}(w)$ properly contained in $A(w)$ for $\mathcal{P}$-a.a. $w$ with

$$
\ell(\mathcal{B}(A)(w))=\ell\left(\mathcal{B}\left(A^{\prime}\right)(w)\right)
$$

for $\mathcal{P}$-a.a. $w$.

One can relate random and non-random attractors using the following result.

Lemma 3.11. If $A$ is a random attractor, then it is a random Milnor attractor.

Proc. R. Soc. Lond. A (1999) 
Proof. Given a random attractor $A$, note that for $\mathcal{P}$-a.a. $w, \mathcal{B}(A)(w)$ contains an open set and, hence, $\ell(\mathcal{B}(A)(w))>0$ on a set of full $\mathcal{P}$-measure. Hence,

$$
l(\operatorname{graph}(\mathcal{B}(A)))=\int \ell(\mathcal{B}(A(w))) \mathrm{d} \mathcal{P}(w)>0 .
$$

Lemma 3.12. Suppose that $A$ is a random invariant set; then

$$
\mathcal{P}(\{w: \mathcal{B}(\overline{\operatorname{graph}(A)})(w) \neq \mathcal{B}(\tilde{A})(w)\})=0 .
$$

Proof. Observe that

$$
\begin{aligned}
\mathcal{B}(\overline{\operatorname{graph}(A)}) & =\left\{(w, x): \lim _{n \rightarrow \infty} d\left(F^{n}(w, x), \overline{\operatorname{graph}(A)}\right)=0\right\} \\
& =\left\{(w, x): \lim _{n \rightarrow \infty} d\left(\left(\theta^{n}(w), \phi(n, w) x\right), \overline{\operatorname{graph}(A)}\right)=0\right\} \\
& =\left\{\begin{array}{c}
\lim _{n \rightarrow \infty} d_{W}\left(\theta^{n}(w), \operatorname{supp}(\mathcal{P})\right)=0, \\
(w, x): \text { and } \\
\lim _{n \rightarrow \infty} d_{X}\left(\phi(n, w) x, \tilde{A}\left(\theta^{n}(w)\right)\right)=0 .
\end{array}\right.
\end{aligned}
$$

But for $\mathcal{P}$-almost all $w$, we have $d_{W}\left(\theta^{n}(w), \operatorname{supp}(\mathcal{P})\right)=0$, and so, for $\mathcal{P}$-a.a. $w$, we have $(w, x) \in \mathcal{B}(\overline{\operatorname{graph}(A)})$ if and only if $x \in \mathcal{B}(\tilde{A})(w)$.

Theorem 3.13. Suppose that $A$ is a random invariant set. Then $\tilde{A}$ is a random Milnor attractor if and only if $\overline{\operatorname{graph}(A)}$ is a Milnor attractor.

Proof. Lemma 3.12 implies that

$$
l(\mathcal{B}(\operatorname{graph}(\tilde{A})))=\int \ell(\mathcal{B}(\tilde{A})(w)) \mathrm{d} \mathcal{P}(w) .
$$

This implies that, if $\tilde{A}$ is a random Milnor attractor, then $\overline{\operatorname{graph}(A)}$ is a Milnor attractor. Conversely, if $l(\mathcal{B}(\overline{\operatorname{graph}(A)}))>0$, then there must be a $\mathcal{P}$-positive measure set of $w$ such that $\ell(\mathcal{B}(\tilde{A}(w)))>0$. Let

$$
Q=\{w: \ell(\mathcal{B}(\tilde{A})(w))=0\} .
$$

Recall that $\mathcal{B}(\operatorname{graph}(A))$ is $F$-invariant, and $F$ is a homeomorphism. Hence, $F$ maps sets of positive $l$-measure on fibres to themselves, and so $Q$ must be $\theta$-invariant. Ergodicity of $\mathcal{P}$ implies that $Q$ must have zero measure and the result follows.

Remark 3.14. The assumption that $\overline{\operatorname{graph}(A)}$ is compact is necessary for the definition of a Milnor attractor. There are, presumably, generalizations of this definition that will allow for the non-compact attractors.

The next lemma relates minimality of $A$ and $\tilde{A}$.

Lemma 3.15. If $A$ is a minimal random Milnor attractor, then $\overline{\operatorname{graph}(A)}$ is a minimal Milnor attractor.

Proof. This follows because $\tilde{A}$ is the smallest closed set (in the topology on $W \times X$ ) that contains $A$.

Proc. R. Soc. Lond. A (1999) 
Note that $\tilde{A}$ is not necessarily a minimal random Milnor attractor even if $\overline{\operatorname{graph}(A)}$ is a minimal Milnor attractor. Random attractors according to definition 3.5 are random Milnor attractors but the converse is not necessarily true even in the nonrandom case. The examples of riddled basins in Alexander et al. (1992) and Ashwin et al. (1994) show this by constructing attractors whose basins have positive background measure but which contain no open sets. See proposition 5.1 for an explicit example of this.

\section{Random invariant measures}

In $\S 3$ we discussed Milnor attractors for RDSs. In this section we study some invariant measures supported on random attractors and their relation to invariant, and, in particular, natural and inner singular, measures for $F$. Recall that all measures are Borel measures on the appropriate space.

A random probability measure is a measurable map from $W$ to probability measures on $X$. The measure $\mu_{w}$ is said to be invariant if

$$
\psi_{w} \mu_{w}=\mu_{\theta(w)},
$$

for $\mathcal{P}$-almost all $w$. Note that any probability measure $\mu$ on $W \times X$ that is invariant under (1.1) and has marginal $\mathcal{P}$ on $W$ has a measurable decomposition $w \mapsto \mu_{w}$, such that

$$
\mu(\operatorname{graph}(B))=\int_{W} \mu_{w}(B(w)) \mathrm{d} \mathcal{P}(w),
$$

for any Borel random set $B(w)$ (see, for example, Crauel \& Flandoli 1994; Crauel 1995). This decomposition $\mu_{w}$ is $\mathcal{P}$-a.e. uniquely defined. Observe that the correspondence $\mu \rightarrow \mu_{w}$ is a map from the subset of $\operatorname{Pr}(W \times X)$ with marginal $\mathcal{P}$ to measurable maps $W \rightarrow \operatorname{Pr}(X)$. The random support of $\mu_{w}$ is the random set that is the support of $\mu_{w}$ in each fibre.

Remark 4.1. If $\mu_{w}$ is a random invariant measure with random $\operatorname{support} \operatorname{supp}\left(\mu_{w}\right)$ then the invariant measure $\mu$ has support $\operatorname{graph}\left(\operatorname{supp}\left(\mu_{w}\right)\right)$.

Definition 4.2. If $\mu$ is a natural measure for $F$, then we call its decomposition, $\mu_{w}$, a random natural measure.

Remark 4.3. It is possible, presumably, to define the concept of a random generic set and, hence, random natural measure by looking at the attraction of random sets; however, we have not found an elegant way of doing this in a pointwise way analogous to the definition of random Milnor attractors, and so we do not attempt to discuss this in any generality.

If we have a random Milnor attractor that is a random point set, $A(w)=\{a(w)\}$, then the only probability measure that can be supported on $A$ is the random $\delta$ function $\delta_{a(w)}$. In this case, it follows that $\delta_{a(w)}$ must be a random natural measure on the attractor. If such an $A(w)$ is invariant, we say $A$ is a random fixed point. Note that a random fixed point is exactly invariant.

(a) Inner singular measures for RDSs

Schenk-Hoppé (1996b) discussed random invariant measures that are unstable, i.e. not attracting in the sense that their supports are not attractors. We consider

Proc. R. Soc. Lond. A (1999) 
invariant measures that are singular not only on a set of $w$ of full $\mathcal{P}$-measure but also for $w \in \operatorname{supp}(\mathcal{P})$. One can use lemma 2.3 to obtain the following result.

Lemma 4.4. Suppose that $\mu_{w}$ is a random natural measure for $F$; then any initial point $(w, x)$ that is generic for this measure will have

$$
\mathcal{E}_{\mu} \subseteq \tilde{\omega}_{\text {meas }}(w, x) .
$$

In other words, any trajectory attracted to this natural measure will display arbitrarily long orbit segments that show behaviour typical for another measure that may not even have marginal $\mathcal{P}$. As an example, suppose we force a system by unbiased i.i.d. noise; then we will be able to see arbitrarily long orbit segments where the noise appears to be biased. Furthermore, given any different distribution, there will be arbitrary long segments that appear as though that distribution is governing the noise.

If $\mathcal{P}$ is chaotic (i.e. there are inner singular measures $\mathcal{Q}$ for the transformation $\theta$ with respect to $\mathcal{P}$ ), one can immediately conclude that there are inner singular measures $\mathcal{E}(\mu)$ for $\mu$. This is a simple observation, but it says a lot about the structure of random attractors. For example, it will affect uniformity of attraction and continuity of $A(w)$.

\section{Multiplicative noise and the blowout bifurcation}

Now suppose we have a multiplicative $R D S$, i.e.

$$
F(w, x)=\left(\theta(w), \psi_{w}(x)\right),
$$

where

$$
\psi_{w}(x)=\Psi_{w} x+O\left(x^{2}\right),
$$

and $\Psi_{w}$ is a linear map on $\boldsymbol{R}^{n}$. It can be seen that $F$ has a random fixed point at $x=0$ and that for fixed $n$

$$
\phi(n, w) x=\Phi(n, w) x+O\left(x^{2}\right),
$$

where $\Phi(n, w)$ is a linear cocycle. Note that in this case, 0 has been called a 'deterministic' fixed point by, for example, Arnold et al. (1996); however, note that the linearization at the fixed point will still fluctuate with $w$ and so we prefer to avoid this terminology. By considering the spectrum of Lyapunov exponents (LEs) of inner singular invariant measures, one can classify attractors and bifurcations of such systems.

The rest of this section is as follows; we discuss Lyapunov exponents in general and then concentrate on a particular example of multiplicative noise. We show how Lyapunov exponents can be used to determine whether a trivial random fixed point is an attractor, and how it may lose stability.

\section{(a) Spectrum of Lyapunov exponents}

Assume that the matrix valued function $\Psi_{w}$ and its inverse are bounded uniformly in $w$ and $x$; this is typical for smooth invertible maps, and is sufficient, though not necessary, for what follows. We assume, moreover, that $\log \left\|\Psi_{w}\right\|^{ \pm 1}$ is uniformly bounded above and below. For $w \in W$ and $|v|=1$, define

$$
\lambda(w, v)=\lim _{n \rightarrow \infty}(1 / n) \log \|\Phi(n, w) v\|,
$$

Proc. R. Soc. Lond. A (1999) 
the Lyapunov exponent in the direction $v$, if this limit exists. By Oseledec's theorem (Oseledec 1968), given any ergodic measure $\mu$ for $F$, this limit is equal to one of a finite number of possible values for $\mu$-almost all $(w, v) \in W \times X$; we define these to be

$$
\lambda_{1}(\mu)>\lambda_{2}>\cdots>\lambda_{d}(\mu),
$$

the set of Lyapunov exponents of the random matrix product along the orbits of $\mu$-almost all initial $(w, v)$. If $\theta$ is differentiable (as often studied in the skew-product case), there are tangential LEs defined for $\theta$; hence, the $\lambda_{i}$ are sometimes referred to as the transverse or conditional Lyapunov exponents; we refer to them as fibre LEs. Define

$$
\Lambda(\mu)=\lambda_{1}(\mu), \quad \lambda(\mu)=\lambda_{d}(\mu)
$$

and then

$$
\Lambda_{\max }(\mu)=\sup _{\nu \in \mathcal{E}_{\mu}} \Lambda(\nu) .
$$

Note that

$$
\Lambda(\mu) \leqslant \Lambda_{\max }(\mu),
$$

and that $\Lambda_{\max }$ is dependent on the inner singular measures with respect to $\mu$.

Moreover, one can generically expect strict inequality in (5.2) for chaotic invariant measures $\mu$ (see Ashwin et al. 1996). If $\theta$ is chaotic, this can cause large deviation phenomena in the convergence of the LEs; effects of this have been studied for example by Pikovsky \& Grassberger (1991) and Baxendale (1994).

\section{(b) Case study for multiplicative noise}

We now concentrate on a particular example system that models forcing of a onedimensional invertible map by an i.i.d. process; such and more general settings are often used to model perturbation by noise (see, for example, Kifer 1988; Stark et al. 1997).

Suppose that $W=\{0,1\}^{Z}$, the space of bi-infinite sequences of zeros and ones. We write $w=\left(\ldots, w_{-1}, w_{0}, w_{1}, \ldots\right)$ and note that this is a metric space with the metric

$$
d(w, v)=\sum_{n \in \boldsymbol{Z}}\left|w_{n}-v_{n}\right| 2^{-|n|} .
$$

Let

$$
\theta: W \rightarrow W, \quad \theta\left(\ldots, w_{-1}, w_{0}, w_{1}, \ldots\right)=\left(\ldots, w_{0}, w_{1}, w_{2}, \ldots\right),
$$

be the shift on $W$ and $\mathcal{P}$ be the Bernoulli measure $B\left(\frac{1}{2}, \frac{1}{2}\right)$; this is ergodic for $\theta$. Let $X=\boldsymbol{R}$ and consider the RDS (1.1) where

$$
\psi_{w}(x)= \begin{cases}x \exp \left(h_{0}\left(x^{2}\right)\right), & \text { if } w_{0}=0, \\ x \exp \left(h_{1}\left(x^{2}\right)\right), & \text { if } w_{0}=1,\end{cases}
$$

and $h_{i}: \boldsymbol{R}^{+} \rightarrow \boldsymbol{R}$ such that $\psi_{w}$ is a homeomorphism for all $w$. This RDS satisfies the hypotheses (H1)-(H3).

For any such $h_{i}(x)$, there is a random invariant measure supported on 0 , i.e. $\delta_{0}$. The fibre LEs can be calculated as

$$
\Lambda=\frac{1}{2}\left(h_{0}(0)+h_{1}(0)\right), \quad \Lambda_{\max }=\max \left(h_{0}(0), h_{1}(0)\right) .
$$

In this context, the following result is elementary to prove.

Proc. R. Soc. Lond. A (1999) 
Proposition 5.1. For this system and the random ergodic measure $\delta_{0}$

(i) $\Lambda_{\max }<0$ implies uniform stability of 0 ;

(ii) $\Lambda<0$ implies 0 is a random Milnor attractor; and

(iii) $\Lambda>0$ implies that 0 is not an attractor.

Thus, in case (i) or (ii), $\delta_{0}$ is a random natural measure.

Proof. (i) Suppose that $h_{i}(0)<\gamma<0$. Choose $\epsilon>0$ such that $\left.h_{i}\left(x_{0}\right)^{2}\right)<\frac{1}{2} \gamma$ for $\left|x_{0}\right|<\epsilon$. Given any $w \in W$ and $x_{0} \in[-\epsilon, \epsilon]$ by continuity of $h$ we have

$$
\left|\phi(n, w) x_{n}\right|<x_{0} \exp \left(n \frac{1}{2} \gamma\right) \rightarrow 0
$$

as $n \rightarrow \infty$ showing that the non-random neighbourhood $[-\epsilon, \epsilon]$ is contained in the random domain of 0 .

(ii) Now assume that $\frac{1}{2}\left(h_{0}(0)+h_{1}(0)\right)<\gamma<0$. We can find $\epsilon>0$ such that $\frac{1}{2}\left(h_{0}\left(x^{2}\right)+h_{1}\left(x^{2}\right)\right)<\frac{1}{2} \gamma$ for all $|x|<\epsilon$. Let $H=\sup _{0<x<\epsilon} \max \left\{h_{0}(x), h_{1}(x)\right\}$. From the definition of $\mathcal{P}$, for $\mathcal{P}$-a.a. $w$, we have

$$
\lim _{n \rightarrow \infty} \frac{1}{n} \sum_{i=0}^{n-1} w_{i}=\frac{1}{2}
$$

and so for almost all $w$, any $\delta>0$, we can find $N$ with

$$
\frac{1}{n} \sum_{i=0}^{n-1} h_{w_{i}}\left(x_{i}\right)<(1-\delta) \gamma+\delta H,
$$

for $n \geqslant N$, as long as $0<x_{i}<\epsilon$ for all $i=0 \cdots n-1$. Choose $\delta$ so that the right-hand side is less than some $\xi<0$. Now

$$
\left|x_{n}\right|=\left|\phi(n, w) x_{0}\right|=\left|x_{0} \exp \left(\sum_{0}^{n-1} h_{w_{i}}\left(x_{i}\right)\right)\right|<x_{0} \exp (n \xi),
$$

with the final inequality holding for $n>N$, as long as $|\phi(n, w) x|<\epsilon$ for $0<n<N$. By continuity of $\phi$, we can conclude that there is an $\eta(w)>0$ such that if $\left|x_{0}\right|<\eta(w)$, then $\left|x_{n}\right|<\epsilon$ for $0<n<N$, and so we conclude that

$$
\lim _{n \rightarrow \infty} \phi(n, w) x=0
$$

for all $x$ with $|x|<\eta(w)$, where $\eta(w)>0$ for $\mathcal{P}$-a.a. $w$. This means that 0 is a random Milnor attractor.

(iii) This can be proved in a similar way to (ii) by showing that there is an $\epsilon>0$ such that for $\mathcal{P}$-a.a. $w$ and any $x \neq 0,|\phi(n, w) x|>\epsilon$ for all large enough $n$.

Remark 5.2. Similar results hold for many maps, even if they are not skew products. All that is really necessary is the invariant subsystem, in this case the subsystem where $x=0$. The result (ii) above for $C^{1+\alpha}$ maps can be shown using Pesin theory (see Alexander et al. 1992); the results (i) and (iii) for $C^{1+\alpha}$ maps are shown in Ashwin et al. (1996). Note that without the skew-product structure, the proof is considerably more difficult. Moreover, the above can be observed in numerical simulations for a wider range of systems.

Proc. R. Soc. Lond. A (1999) 
Remark 5.3. A basin of attraction of a Milnor attractor $A$ is locally riddled if there is a neighbourhood $U$ of $A$ such that given any neighbourhood $V$ of $A$, there is a positive measure set of points in $V$ that leave $U$ under iteration. It is globally riddled if any open set intersecting $\mathcal{B}(A)$ also intersects its complement in a set of positive background measure (Alexander et al. 1992). Suppose then that $\Lambda_{\max }>0>\Lambda$ for (5.4). By exploiting the non-uniformity of $\eta(w)$ in (ii) we can show that the basin of attraction of $W \times\{0\}$ for the skew-product system must be locally or even globally riddled.

\section{(c) Branching of random attractors and D-bifurcations}

Suppose we have a family of RDSs for multiplicative noise generated by iterating (1.1) parametrized continuously by some $\alpha \in \boldsymbol{R}$. We write the cocycle (5.1) as

$$
\phi_{\alpha}(n, w) x=\Phi_{\alpha}(n, w) x+O\left(x^{2}\right) .
$$

Assume that $\theta$ is independent of $\alpha$. We say that $\alpha$ is a normal parameter for the subsystem when $x=0$ (Ashwin et al. 1996).

Suppose that (5.1) has a family of random ergodic measures $\mu_{w}(\alpha)$. If $\mu(\alpha)$ varies continuously in $\alpha$ (in the weak-* topology on $\operatorname{Pr}(W \times X)$ ), then we say $\operatorname{supp}\left(\mu_{w}\right)(\alpha)$ is a branch of random ergodic measures. If $\mu_{w}$ are random natural measures, we say $\operatorname{supp}\left(\mu_{w}\right)(\alpha)$ is a branch of random attractors.

Recall that continuity in the weak-* topology implies that if we average any continuous observation from the system, this average will vary continuously with $\alpha$.

Definition 5.4. Suppose that (5.1) has a branch of random ergodic measures $\mu_{w}(\alpha)$ and that these support random attractors if and only if $\alpha<\alpha_{0}$. We say the branch loses stability at $\alpha_{0}$.

If two (or more) branches of random attractors supporting $\mu_{w}(\alpha)$ and $\nu_{w}(\alpha)$, $\alpha<\alpha_{0}$ are such that $\mu_{w}\left(\alpha_{0}\right)=\nu_{w}\left(\alpha_{0}\right)$ for $\mathcal{P}$-a.a. $w$, we say the branches meet at $\alpha_{0}$.

We refer to a parameter value where a branch loses stability, or where two or more branches meet, as a D-bifurcation of random attractors.

Arnold and co-workers (Arnold 1998; Arnold et al. 1997) discussed several examples of this type of bifurcation; much work has been done to identify and classify D-bifurcations of attractors in systems, and a surprising number of similarities are found with deterministic bifurcation theory (see Baxendale (1994), Arnold \& Boxler (1990), Arnold et al. (1996) and Crauel \& Flandoli (1998) for some examples).

An easy consequence of proposition 5.1 is as follows.

Corollary 5.5. For a parametrized family of systems as in proposition 5.1, if $\Lambda\left(\alpha_{0}\right)=0$ and $\mathrm{d} \Lambda / \mathrm{d} \alpha \neq 0$, then 0 loses stability at a $D$-bifurcation at $\alpha=\alpha_{0}$.

This agrees with Arnold \& Xu (1994), who imply that a necessary condition for Dbifurcation is that a fibre LE is zero. Although fibre LEs for (5.4) will vary smoothly, this is not the case in general. Nevertheless, they will be discontinuous on a set that is small in a certain sense (Arnold \& Cong 1994) and for many parametrized systems, the LEs are continuous on open and dense subsets of parameter space.

Corollary 5.5 can be interpreted as saying that the attractor in the subsystem $x=0$ undergoes a blowout at $\alpha_{0}$. This idea was introduced by Ott \& Sommerer (1994); see also Yamada \& Fujisaka (1984) and Pikovsky (1984).

Proc. R. Soc. Lond. A (1999) 
An interesting (but very difficult) question is what can one say about other random invariant sets, in particular attractors that may branch from 0 .

Remark 5.6. Note that $A_{\alpha}=\operatorname{supp}\left(\mu_{w}(\alpha)\right)$ need not change continuously in the Hausdorff metric if $\mu(\alpha)$ is a branch. At a blowout bifurcation, the dimension of the attractor and $A_{\alpha}$ are observed to change discontinuously with $\alpha$. This originally suggested the name 'blowout'. The next subsection illustrates this point via an example.

\section{(d) Case study of a branched attractor}

We consider the set-up as in $\S 5 b$ with

$$
\psi_{w}(x)= \begin{cases}\alpha x, & \text { if } w_{0}=0, \\ \beta x \exp (h(x)), & \text { if } w_{0}=1,\end{cases}
$$

where $0<\alpha<1<1 / \alpha<\beta$ and $h(0)=0, h(1)=-\log \beta, h^{\prime}(0)=0$ and $-2<h^{\prime}(x)<0$ for all $x>0$. In this case (which clearly falls into case (iii) of proposition 5.1) we know that 0 is not a random attractor. We show that there is, however, a unique random attractor in $x \geqslant 0$ and prove some properties of it.

Theorem 5.7. For the above system, there is a unique random fixed-point attractor $A(w)$ in $x \geqslant 0$ such that

(i) For $\mathcal{P}$-a.a. $w$

$$
\limsup _{n \rightarrow \infty} \phi(n, w) A(w)>0 \quad \text { and } \quad \liminf _{n \rightarrow \infty} \phi(n, w) A(w)=0 ;
$$

(ii) if it exists, the fibre LE must be non-positive;

(iii) for any open $U \subset W$,

$$
\operatorname{ess} \sup _{w \in U} A(w)>0 \quad \text { and } \quad \operatorname{ess}_{\inf } \tilde{w}_{\in U} A(w)=0 ;
$$

(iv) $\mathcal{B}(A)(w)=(0, \infty)$.

In other words, $A(w)$ is a uniformly stable fixed-point random attractor that is discontinuous at $\mathcal{P}$-almost all $w$.

Proof. The assumptions on $\psi$ imply that for any $w$ and $x>1, \psi_{w}(x)<x$, and so all asymptotic dynamics must be contained in $[0,1]$. We change coordinates by setting $\xi=\log (x)$; write $x_{n}=\phi(n, w) x_{0}$. The transformation on $\boldsymbol{R}^{+}$in these coordinates is $\xi_{1}=\Psi_{w} \xi_{0}$, where $\Psi_{w}(\xi)=\log \left(\psi_{w}\left(\mathrm{e}^{\xi}\right)\right)$. Similarly we write $\xi_{n}=\Phi(n, w)\left(\xi_{0}\right)=$ $\log \phi(n, w) \mathrm{e}^{\xi_{0}}$. Note that

$$
\left|\Psi_{0}^{\prime}(\xi)\right|=1 \quad \text { and } \quad\left|\Psi_{1}^{\prime}(\xi)\right|=\left|1+h^{\prime}\left(\mathrm{e}^{\xi}\right) \mathrm{e}^{\xi}\right|<1, \quad \text { for all } \xi \leqslant 0,
$$

where we have used the assumption that $h^{\prime}\left(\mathrm{e}^{\xi}\right)>-2$. Hence,

$$
\left|\xi_{n}-\eta_{n}\right|=\left|\int_{\eta_{0}}^{\xi_{0}} \Psi_{w_{n-1}}^{\prime}\left(s_{n-1}\right) \cdots \Psi_{w_{0}}^{\prime}\left(s_{0}\right) \mathrm{d} s_{0}\right| \leqslant\left|\xi_{0}-\eta_{0}\right|,
$$

Proc. R. Soc. Lond. A (1999) 
for all $w, n \geqslant 1$. Since, by (iii) of proposition 5.1, we know that for $\mathcal{P}$-a.a. $w$, $\lim \sup x_{n}>0$ and so $\left|\xi_{n}-\eta_{n}\right| \rightarrow 0$ as $n \rightarrow \infty$. Hence, for $\left|x_{n}-y_{n}\right| \rightarrow 0$ as $n \rightarrow \infty$ for any initial conditions $x_{0}, y_{0}>0$ and any $w$. Now pick an arbitrary $x_{0}>0$ and let

$$
a_{n}(w)=\phi\left(n, \theta^{-n} w\right) x_{0} .
$$

By arguments in Crauel \& Flandoli (1998) using the monotonicity of $\psi$, this converges almost surely to some invariant $A(w)$ as $n \rightarrow \infty$ and this limit is independent of $x_{0}$.

Moreover, $\lim _{n \rightarrow \infty}\left|x_{n}-\phi(n, w) A(w)\right|=0$ for any $x_{0}>0$ and so (iv) holds. Examining

$$
\lambda=\limsup _{n \rightarrow \infty}(1 / n) \phi^{\prime}(n, w) x,
$$

this is clearly non-positive, implying (ii). Now pick any $w$ and consider $n_{j}$ such that

$$
\sum_{k=n_{j}}^{n_{j}+j}\left|w_{k}\right|=0 .
$$

Then $x_{n_{j}+k}=\alpha^{k} x_{n_{j}}<\alpha^{k}$ for $k=0, \ldots, j$. Almost all $w$ will contain such sequences for all $j$, and so we can conclude (i).

To show (iii), let

$$
V=\left\{v: v_{i}=w_{i}, \text { for all }|i|<N\right\} .
$$

Pick an $N$ such that $V \subset U$. Let

$$
V^{m}=\left\{v \in V: v_{i}=0, \text { for all }|i|=N+1, \ldots, N+m\right\} .
$$

These are open sets where $\phi\left(n, \theta^{-n} w\right) x_{0}<\alpha^{m} \beta^{N}$ for all $n>N=M$. By choosing $m$ large, we see that (iii) holds.

The problem is to construct an invariant graph, and, in this aspect, the above result is similar to the proof of existence of invariant graphs of Stark (1997) and Keller (1996). Note that it is relatively easy to show that for any $x_{0}$ and $y_{0},\left|x_{n}-y_{n}\right| \rightarrow 0$. Note also that for this example, the convergence $d_{X}\left(\phi(n, w) x, A\left(\theta^{n} w\right)\right) \rightarrow 0$ is almost sure, as required for our definition of a random basin of attraction. Observe also that although the transformation is continuous, the random attractor we obtain is discontinuous in a rather extreme way.

We do not construct invariant measures on $A(w)$, but note that this can presumably be performed using the techniques of Crauel \& Flandoli (1998).

\section{(e) D-bifurcation scenarios}

As suggested by studies of blowout bifurcations, there are at least two possible 'generic' scenarios at bifurcation, the hysteretic and non-hysteretic scenarios of Ott \& Sommerer (1994). Roughly speaking, they are analogous to subcritical and supercritical bifurcations of fixed points. However, unlike equilibrium bifurcations, there is no hope of determining criticality by looking at truncated Taylor expansions of the cocycle near 0 . This is because both before and after a blowout bifurcation, the attractor will be embedded in an invariant set that has finite size deviations away from 0 . The two scenarios of Ott \& Sommerer are as follows.

Proc. R. Soc. Lond. A (1999) 
(1) Hysteretic: in this case, there is no other branch of attractors that converges to $\delta_{0}$. After the blowout, almost all trajectories leave a neighbourhood of the attractor never to return. Before the blowout, the basin of 0 is riddled (see remark 5.3).

(2) Non-hysteretic: in this case, the stability of $\delta_{0}$ is transferred to one or more branches of on-off intermittent states that limit to $\delta_{0}$ (see Platt et al. 1993). Before the blowout, the basin is locally riddled but not globally riddled. This means that a typical trajectory $\left(w_{n}, x_{n}\right)$ will be such that $x_{n}$ makes finite size deviations infinitely often, but also given any $\delta>0,\left|x_{n}\right|<\delta$ for arbitrarily long periods. The mean $\langle|x|\rangle$ along a trajectory exists and is typically positive.

Note that the stochastic Hopf bifurcations investigated by Baxendale (1994) are forced to be non-hysteretic by hypothesis, as are the bifurcations studied in Arnold et al. (1996). Moreover, there are other possible generic bifurcations, especially if $\phi$ commutes with a group action on $X$ (see, for example, Ashwin 1997).

One open problem is to classify the scenario of a generic unfolding from properties of the system at bifurcation. Ashwin et al. (1998) suggest that criticality may be related to the presence of an essential basin of attraction at the bifurcation point. This is a positive background measure set of points that limit to the subsystem $x=0$ in a rather weaker sense that averages of observables on trajectories are determined only by values of the observables on $x=0$.

Remark 5.8. Another issue that we do not consider here is that of two-point stability of random attractors, i.e. given nearby initial conditions in the same fibre, whether their difference goes to zero or not. One-dimensional invertible systems of the form (5.4) will always have attractors that are two-point stable; this is because their fibre LEs are forced to be negative. However, for non-invertible or two- or more-dimensional systems, a random attractor may or may not be two-point stable (Baxendale 1998). Ashwin et al. (1998) compute a supercritical blowout in a simple piecewise linear (non-invertible) skew-product map and show that the fibre LEs on the bifurcating branches may be negative (giving a random attractor that is a graph) or positive (giving an attractor for the skew-product system that is 'hyperchaotic').

\section{Discussion}

In conclusion, we show that there are close parallels between definitions and results for (a) attractors for RDSs and Milnor attractors of deterministic dynamical systems; and (b) D-bifurcations of multiplicative noise RDSs and blowout bifurcations of deterministic dynamical systems with invariant subsystems. We emphasize the role of minimal attractors and show how a random Milnor attractor generalizes the idea of a random attractor.

It should be profitable to exploit the similarities between RDSs and deterministic dynamical systems; for example, the supercritical and subcritical scenarios of branching of attractors in blowouts must also be present in RDSs. Likewise, the fact that blowout bifurcations are usually considered to occur in finite-dimensional deterministic systems is an unnecessary restriction; they will occur in quite general systems. Also, the work on RDSs should be able to illuminate the structure of on-off intermittent attractors.

Proc. R. Soc. Lond. A (1999) 
Work from blowout bifurcations suggests that there are several possible scenarios at loss of stability of a random attractor. There may or may not be nearby branches of attractors and these branches may or may not be stable for two-point motion. It is an open problem to formulate testable conditions that determine which scenario will appear in a given system.

Blowout problems provide examples of deterministic systems where Milnor's notion of an attractor comes into its own, i.e. examples of attractors whose basins are riddled. Since riddled basins are known to be destroyed by arbitrary small noise perturbations, it would be of interest to look at invariant measures and attractors for randomly forced systems showing riddled basins in the unforced case. One surprising result that needs to be better understood is why the branching at blowout bifurcation seems to be relatively robust even to perturbations that break the skew-product structure (see, for example, Ashwin 1995).

The main assumption made here on the structure of RDSs is that the base transformation is continuous on a compact space and that the RDS is continuous in this base variable; this is very important to give structure to the random attractors. It is a challenge to see if this could be weakened to give information about stochastic differential equations (SDEs) that do not presently fit into this framework. Nevertheless, some schemes for numerical approximation of SDEs do fit into the presented framework.

I would like to thank the following people for very interesting conversations: K. Schenk-Hoppé, N. Sri Namachchivaya, P. Baxendale and M. Nicol. I also thank H. Crauel for his very insightful comments on the first draft of this paper and suggesting the term 'inner singular measure'. A Nuffield 'Newly appointed science lecturer' grant enabled discussion with the aforementioned researchers. I am grateful to the referees for their advice and comments.

\section{References}

Alexander, J. C., Kan, I., Yorke, J. A. \& You, Z. 1992 Riddled basins. Int. J. Bifurcation Chaos 2, 795-813.

Arnold, L. 1998 Random dynamical systems. Springer Monographs in Mathematics. Berlin: Springer.

Arnold, L. \& Boxler, P. 1990 Stochastic bifurcation: instructive examples in dimension one. In Stochastic flows. Proceedings of Charlotte, North Carolina 1990 (ed. M. Pinsky \& V. Wihstutz), pp. 241-255. Basel: Birkhäuser.

Arnold, L. \& Cong, N. D. 1994 Generic properties of Lyapunov exponents. Random Computational Dynamics 2, 335-345.

Arnold, L. \& Xu, K. 1994 Invariant measures for random dynamical systems, a necessary condition for stochastic bifurcation from a fixed point. Random Computational Dynamics 2, $165-182$.

Arnold, L., Sri Namachchivaya, N. \& Schenk-Hoppé, K. R. 1996 Toward an understanding of stochastic Hopf bifurcation: a case study. Int. J. Bifurcation Chaos 6, 1947-1975.

Ashwin, P. 1995 Cycles homoclinic to chaotic invariant sets; robustness and resonance. Phys. Lett. A 209, 338-344.

Ashwin, P. 1997 Cycles homoclinic to chaotic invariant sets; robustness and resonance. Chaos 7, 207-220.

Ashwin, P., Buescu, J. \& Stewart, I. N. 1994 Bubbling of attractors and synchronisation of chaotic oscillators. Phys. Lett. A 193, 126-139.

Proc. R. Soc. Lond. A (1999) 
Ashwin, P., Buescu, J. \& Stewart, I. N. 1996 From attractor to chaotic saddle: a tale of transverse instability. Nonlinearity 9, 703-737.

Ashwin, P., Aston, P. \& Nicol, M. 1998 On the unfolding of a blowout bifurcation. Physica D 111, 81-95.

Baxendale, P. 1994 A stochastic Hopf bifurcation. Prob. Theory Relat. Fields 99, 581-616.

Baxendale, P. 1998 Stability along trajectories at a stochastic bifurcation point. Preprint.

Crauel, H. 1995 Random probability measures on Polish spaces. Habilitationsschrift, University of Bremen.

Crauel, H. \& Flandoli, F. 1994 Attractors for random dynamical systems. Prob. Theory Related Fields 100, 365-393.

Crauel, H. \& Flandoli, F. 1998 Additive noise destroys a pitchfork bifurcation. J. Dynam. Diff. Eqns 10, 259-274.

Dudley, R. M. 1987 Real analysis and probability. Pacific Grove: Wadsworth \& Brooks/Cole.

Eckmann, J. P. \& Ruelle, D. 1985 Ergodic theory of chaos and strange attractors. Rev. Mod. Phys. 57, 617-656.

Falconer, K. 1997 Techniques in fractal geometry. Chichester: Wiley.

Katok, A. \& Hasselblatt, B. 1995 Introduction to the modern theory of dynamical systems. Cambridge University Press.

Keller, G. 1996 A note on strange nonchaotic attractors. Fundmanta Mathematicae 151, 139148.

Kifer, Y. 1988 Random perturbations of dynamical systems. Progress in Probability and Statistics. Basel: Birkhäuser.

Milnor, J. 1985 On the concept of attractor. Commun. Math. Phys. 99, 177-195. (Comments (1985) Commun. Math. Phys. 102, 517-519.)

Mora, L. \& Viana, M. 1993 Abundance of strange attractors. Acta Math. 171, 1-71.

Oseledec, V. I. 1968 A multiplicative ergodic theorem: Lyapunov characteristic numbers for dynamical systems. Trans. Mosc. Math. Soc. 19, 197-231.

Ott, E. \& Sommerer, J. C. 1994 Blowout bifurcations: the occurrence of riddled basins and on-off intermittency. Phys. Lett. A 188, 39-47.

Pikovsky, A. S. 1984 On the interaction of strange attractors. Z. Phys. B 55, 149-154.

Pikovsky, A. S. \& Grassberger, P. 1991 Symmetry breaking bifurcation for coupled chaotic attractors. J. Phys. A 24, 4587-4597.

Platt, N., Spiegel, E. A. \& Tresser, C. 1993 On-off intermittency; a mechanism for bursting. Phys. Rev. Lett. 70, 279-282.

Ruelle, D. 1989 Chaotic evolution and strange attractors. Cambridge University Press.

Schenk-Hoppé, K. R. 1996 a Random attractors. I. Discussion paper 327, Department of Economics, University of Bielefeld, Germany.

Schenk-Hoppé, K. R. $1996 b$ Random attractors. II. Discussion paper 327, Department of Economics, University of Bielefeld, Germany.

Stark, J. 1997 Invariant graphs for forced systems. Physica D 109, 163-179.

Stark, J., Broomhead, D. S., Davies, M. E. \& Huke, J. 1997 Takens embedding theorems for forced and stochastic systems. Nonlinear Analysis 30, 5303-5314.

Yamada, T. \& Fujisaka, H. 1984 Stability theory of synchronised motion in coupled-oscillator systems. Prog. Theor. Phys. 70, 1240-1248.

Proc. R. Soc. Lond. A (1999) 